\title{
Precision Measurement of the Proton Flux in Primary Cosmic Rays from 1 GV to 1.8 TV with the Alpha Magnetic Spectrometer on the International Space Station.
}

\author{
Vitali Choutko* \\ Massachusetts Institute of Technology, MIT, Cambridge, MA 02139, USA \\ E-mail: vitali.choutkodcern.ch
}

\begin{abstract}
A precision measurement of the proton flux in primary cosmic rays with rigidity $1 \mathrm{GV}$ to $1.8 \mathrm{TV}$ is presented based on 300 million events. The results show that proton flux is smooth and exhibits no sharp structures with rigidity. The detailed variation with rigidity of the flux spectral index is presented for the first time. The spectral index is progressively hardening at high rigidities.
\end{abstract}

The 34th International Cosmic Ray Conference,

30 July- 6 August, 2015

The Hague, The Netherlands

\footnotetext{
* Speaker.
} 


\section{Introduction}

Protons are the most abundant particles in cosmic rays. Knowledge of the precise behavior of the proton flux with energy is important in understanding the origin, acceleration, and propagation of cosmic rays [1]. The flux of protons in cosmic rays has been previously reported [2, 3, 6, 4, 5]. Here we report on the precise measurement of the proton flux in the rigidity range from $1 \mathrm{GV}$ to 1.8 TV performed with 41 billion events collected by the Alpha Magnetic Spectrometer (AMS) during the first 30 months of operation onboard the International Space Station (ISS).

\section{Detector}

AMS is a general purpose high energy particle physics detector in space. The layout and description of the detector are presented in Ref. [7]. The key elements used in this measurement are the permanent magnet, the silicon tracker, four planes of time of flight (TOF) scintillation counters, and the array of anticoincidence counters (ACC). AMS also contains a transition radiation detector (TRD), a ring imaging Čerenkov detector (RICH), and an electromagnetic calorimeter (ECAL). The 3-dimensional imaging capability of the 17 radiation length ECAL allows for an accurate measurement of the $e^{ \pm}$energy $E$ and of the shower shape.

The central field of the magnet [8] is $1.4 \mathrm{kG}$. Before flight, the field was measured in 120,000 locations to an accuracy of better than $2 \mathrm{G}$. On orbit the magnet temperature varies from -3 to $+15^{\circ} \mathrm{C}$. The field strength is corrected with a measured temperature dependence of $-0.09 \% /{ }^{\circ} \mathrm{C}$.

The tracker [9] has nine layers, the first (L1) at the top of the detector, the second (L2) just above the magnet, six (L3 to L8) within the bore of the magnet, and the last (L9) just above the ECAL. L2 to L8 constitute the inner tracker. The tracker accurately determines the trajectory of cosmic rays by multiple measurements of the coordinates with a resolution in each layer of $10 \mu \mathrm{m}$ in the bending direction. Together, the tracker and the magnet measure the rigidity $R$ of charged cosmic rays. The maximum detectable rigidity (MDR) is $2 \mathrm{TV}$ over the $3 \mathrm{~m}$ lever arm from L1 to L9.

Each layer of the tracker also provides an independent measurement of the absolute value of the charge $|Z|$ of the cosmic ray. The charge resolution of the layers of the inner tracker together is $\Delta Z \simeq 0.05$ for $|Z|=1$ particles.

Two planes of TOF counters [10] are located above L2 and two planes are located below the magnet. The average time resolution of each counter for $|Z|=1$ particles has been measured to be $160 \mathrm{ps}$ and the overall velocity $(\beta=v / c)$ resolution to be $\Delta \beta / \beta^{2}=4 \%$. This discriminates between upward- and downward-going particles.

The coincidence of signals from the four TOF planes together with the absence of signals from the ACC provides a charged particle trigger. The coincidence of 3 out of the 4 TOF layers with no ACC requirement was used to provide an unbiased trigger. The unbiased trigger, prescaled by $1 \%$, was used to measure the efficiency of the charged particle trigger.

Before launch, at the CERN SPS, AMS was extensively calibrated with 180 and $400 \mathrm{GeV} / c$ proton beams and beams of positrons, electrons, and pions from 10 to $290 \mathrm{GeV} / c$. These data allow the determination of the tracker rigidity resolution function with high precision and the verification of the absolute rigidity scale. Monte Carlo simulated events were produced using a dedicated 
program developed by the collaboration based on the GEANT-4.9.6 package [11]. Figure 11a shows a comparison of the inverse rigidity for $400 \mathrm{GeV} / c$ protons from the test beam and the Monte Carlo simulation. As seen, the resolution has a pronounced Gaussian core with non-Gaussian tails of $\sim 5 \%$ and the simulation precisely reproduces the measured resolution including the tails.
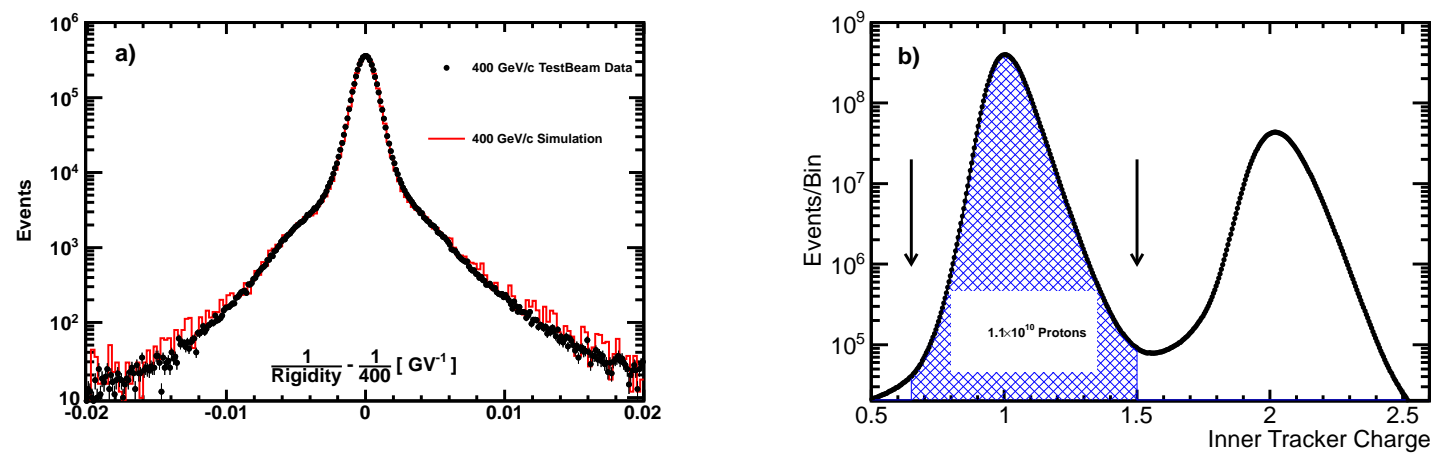

Figure 1: a) The resolution function in inverse rigidity for $400 \mathrm{GeV} / \mathrm{c}$ protons measured in the test beam compared with Monte Carlo simulated events. b) The distribution of the charge $|Z|$ measured by the inner tracker for the cosmic ray events. Protons were selected by requiring $0.65<|Z|<1.5$, as indicated by the arrows.

\section{Selection}

In the first 30 months $\left(7.96 \times 10^{7} \mathrm{~s}\right)$ AMS collected $4.1 \times 10^{10}$ cosmic ray events. Due to the influence of the geomagnetic field, this collection time for primary cosmic rays increases with increasing rigidity becoming constant at $6.29 \times 10^{7} \mathrm{~s}$ above $30 \mathrm{GV}$.

By selecting events to be downward going and to have a reconstructed track in the inner tracker with charge compatible with one we obtain $1.1 \times 10^{10}$ events, see Figure $1 \mathrm{~b}$. In order to have the best resolution at the highest rigidities, further selections are made by requiring the track to pass through L1 and L9 and to satisfy additional track fitting quality criteria such as a $\chi^{2} / d . f .<10$ in the bending coordinate. Then positively charged particles are selected. In addition, to select only primary cosmic rays, well above the geomagnetic cutoff, the measured rigidity is required to be greater than 1.2 times the maximum geomagnetic cutoff within the AMS field of view. The cutoff was calculated by backtracing [12] particles from the top of AMS out to 50 Earth's radii using the IGRF [13] geomagnetic model. These procedures resulted in a sample of $3.0 \times 10^{8}$ primary cosmic rays with $Z=+1$.

Since protons are the dominant component of cosmic rays, the selected sample of $3.0 \times 10^{8}$ events has only small contributions of other particles, mainly deuterons. The deuteron contribution decreases with rigidity; at $1 \mathrm{GV}$ it is less than $2 \%$ and at $20 \mathrm{GV}$ it is $0.6 \%$ [14, 15]. Deuterons were not removed. The sample also contains protons from nuclei which interact at the top of AMS (for example, in L1 or the TRD). From the measured flux [16] and Monte Carlo simulation this contribution is $0.5 \%$ at $1 \mathrm{GV}$ decreasing to less than $0.1 \%$ at and above $10 \mathrm{GV}$. Contamination from $e^{+}$and $e^{-}$[17], overwhelmingly $e^{+}$, was estimated to be less than $0.1 \%$ over the entire rigidity range. The background contributions from protons which originated in the interactions of nuclei 
at the top of AMS and $e^{ \pm}$, both noticeable only below $2 \mathrm{GV}$, are subtracted from the flux and the uncertainties are accounted for in the systematic errors.

\section{Analysis}

The isotropic proton flux $\Phi_{i}$ for the $i^{\text {th }}$ rigidity bin $\left(R_{i}, R_{i}+\Delta R_{i}\right)$ is

$$
\Phi_{i}=\frac{N_{i}}{A_{i} \varepsilon_{i} T_{i} \Delta R_{i}}
$$

where $N_{i}$ is the number of events corrected with the rigidity resolution function (see below), $A_{i}$ is the effective acceptance, $\varepsilon_{i}$ is the trigger efficiency, and $T_{i}$ is the collection time. The proton flux was measured in 72 bins, $i=1$ to 72 , from $1 \mathrm{GV}$ to $1.8 \mathrm{TV}$ with bin widths chosen according to the rigidity resolution. The effective acceptance $A_{i}$ was calculated using Monte Carlo and then corrected for small differences found between the data and Monte Carlo event selection efficiencies. The trigger efficiency $\varepsilon_{i}$ is measured from data with the unbiased trigger events. The trigger efficiency ranges from 90 to $95 \%$. The 5 to $10 \%$ inefficiency is due to secondary $\delta$-rays in the magnetic field entering the ACC. The Monte Carlo agrees with the measured trigger efficiency within $0.5 \%$.

The bin-to-bin migration of events was corrected using a rigidity resolution function obtained from Monte Carlo simulation and verified with the test beam data. The unfolding procedure is described in details in [18].

Extensive studies were made of the systematic errors. The errors include the uncertainties in the trigger efficiency, the acceptance, the background contamination, the geomagnetic cutoff factor, the event selection, the unfolding, the rigidity resolution function, and the absolute rigidity scale. The trigger efficiency error is dominated by the statistics available from the $1 \%$ prescaled unbiased event sample. It is negligible (less than $0.1 \%$ ) below $500 \mathrm{GV}$ and reaches $1.5 \%$ at $1.8 \mathrm{TV}$. The geomagnetic cutoff factor was varied from 1.0 to 1.4 and the resulting proton fluxes showed a systematic uncertainty of $2 \%$ at $1 \mathrm{GV}$ and negligible above $2 \mathrm{GV}$. We have also verified that using the most recent IGRF model [19] and the IGRF model with external non-symmetric magnetic fields [20] does not introduce observable changes in the flux values nor in the systematic errors.

The effective acceptance was corrected for small differences between the data and the Monte Carlo samples related to the event reconstruction and selection. Together, the correction was found to be $5 \%$ at $1 \mathrm{GV}$ decreasing below $2 \%$ above $10 \mathrm{GV}$, while the corresponding systematic uncertainty is less than $1 \%$ above $2 \mathrm{GV}$.

The detector is mostly made of carbon and aluminum. The corresponding inelastic cross sections of $p+\mathrm{C}$ and $p+\mathrm{Al}$ are known to within $10 \%$ at $1 \mathrm{GV}$ and $4 \%$ at $300 \mathrm{GV}$ [21], and $7 \%$ at $1.8 \mathrm{TV}$ from model estimations [11]. The inelastic cross sections are used in the Monte Carlo based estimation of the effective acceptance and, to estimate the systematic error due to the uncertainty in the inelastic cross sections, dedicated samples of protons were simulated with the $p+\mathrm{C}$ and $p+\mathrm{Al}$ cross sections varied by $\pm 10 \%$. From the analysis of these samples together with the current knowledge of the cross sections, a systematic error of $1 \%$ at $1 \mathrm{GV}, 0.6 \%$ from 10 to $300 \mathrm{GV}$, and $0.8 \%$ at $1.8 \mathrm{TV}$ was obtained.

The rigidity resolution function was verified with data from both the ISS and the test beam. For this the residuals between the hit coordinates measured in tracker layers L1 and L9 and those 
obtained from the track fit using the information from only the inner tracker L2 to L8 were compared between data and simulation. In order to validate the alignment of the external layers the difference between the rigidity measured using the information from L1 to L8 and from L2 to L9 was compared between data and the simulation. The resulting uncertainty on the MDR was estimated to be $5 \%$. The corresponding unfolding errors were obtained by varying the width of the Gaussian core of the resolution function by $5 \%$ and the amplitude of the non-Gaussian tails by $\sim 20 \%$ (see for example Fig. 1) over the entire rigidity range and found to be $0.8 \%$ below $200 \mathrm{GV}$ and $3 \%$ at $1.8 \mathrm{TV}$.

There are two contributions to the systematic uncertainty on the rigidity scale. The first is due to residual tracker misalignment. From the $400 \mathrm{GeV} / c$ test beam data it was measured to be less then $1 / 300 \mathrm{TV}^{-1}$. For the ISS data this error was estimated by comparing the $E / p$ ratio for electron and positron events, where $E$ is the energy measured with the ECAL and $p$ is the momentum measured with the tracker, see Ref. [22] for details. It was found to be $1 / 26 \mathrm{TV}^{-1}$, limited by the current high energy positron statistics. The second systematic error on the rigidity scale arises from the magnetic field map measurement $(0.25 \%)$ and its temperature corrections $(0.1 \%)$. Taken in quadrature and weighted by the measured flux, this amounts to less than $0.5 \%$ systematic error on the flux for rigidities above $2 \mathrm{GV}$.

\section{Results}

The measured proton flux is shown in Figure $2 \mathrm{a}$ as a function of rigidity at the top of the AMS detector, including statistical and systematic errors.
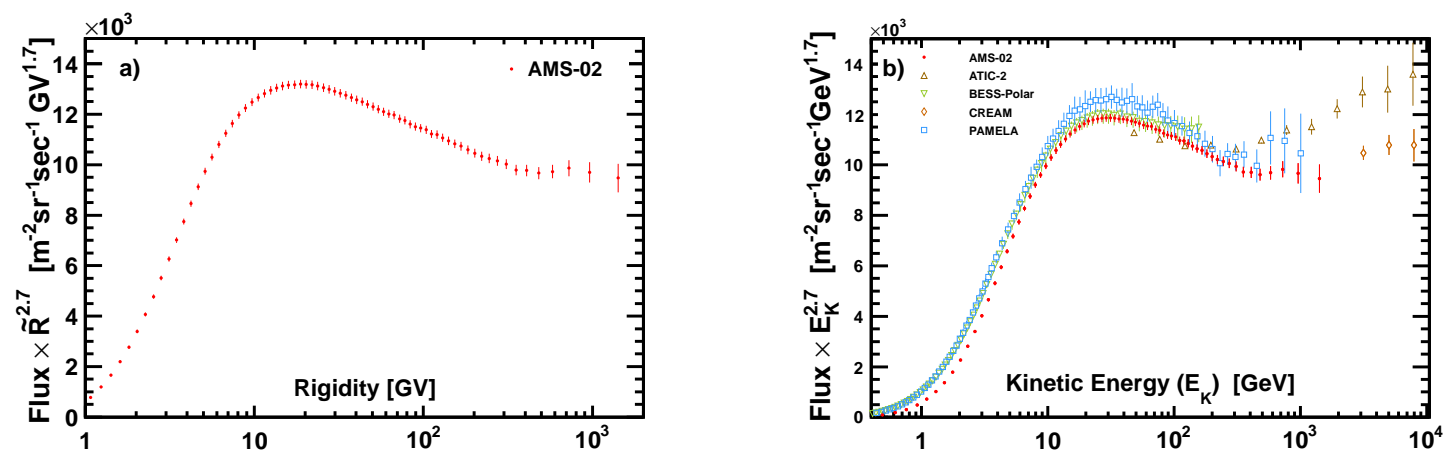

Figure 2: a) The AMS proton flux multiplied by $\tilde{R}^{2.7}$ and total error as a function of rigidity. b) The flux as a function of kinetic energy $E_{\mathrm{K}}$ as multiplied by $E_{\mathrm{K}}^{2.7}$ compared with recent measurements [3, 6, 4, 5]. For the AMS results $E_{\mathrm{K}} \equiv \sqrt{\tilde{R}^{2}+M_{p}^{2}}-M_{p}$ where $M_{p}$ is the proton mass.

The contributions to the systematic errors come from (i) the trigger, (ii) the acceptance, background contamination, geomagnetic cutoff, and event selection, (iii) the rigidity resolution function and unfolding; and (iv) the absolute rigidity scale. The contribution of individual sources to the systematic error are added in quadrature to arrive at the total systematic uncertainty. The Monte Carlo event samples have sufficient statistics such that they do not contribute to the errors. the sum in quadrature of statistical and systematic errors [23]. In this and the subsequent figures, the points 
are placed along the abscissa at $\tilde{R}$ calculated for a flux $\propto R^{-2.7}$ [24]. Figure 2b shows the AMS flux as a function of kinetic energy $E_{\mathrm{K}}$ together with the most recent results (i.e., from experiments after the year 2000).

A power law with a constant spectral index $\gamma$

$$
\Phi=C R^{\gamma}
$$

where $R$ is in GV and $C$ is a normalization factor, does not fit the flux shown in Fig. $2 \mathrm{a}$ at the $99.9 \%$ C.L. for $R>45 \mathrm{GV}$. Applying solar modulation in the force field approximation [25] also does not fit the data at the $99.9 \%$ C.L. for $R>45 \mathrm{GV}$. We therefore fit the flux with a double power law function

$$
\Phi=C\left(\frac{R}{45 \mathrm{GV}}\right)^{\gamma}\left[1+\left(\frac{R}{R_{0}}\right)^{\Delta \gamma / s}\right]^{s}
$$

where $s$ quantifies the smoothness of the transition of the spectral index from $\gamma$ for rigidities below the characteristic transition rigidity $R_{0}$ to $\gamma+\Delta \gamma$ for rigidities above $R_{0}$. Fitting over the range $45 \mathrm{GV}$ to $1.8 \mathrm{TV}$ yields a $\chi^{2} /$ d.f. $=25 / 26$ with $C=0.4544 \pm 0.0004(\mathrm{fit})_{-0.0047}^{+0.0037}(\mathrm{sys})_{-0.0025}^{+0.0027}$ (sol) $\mathrm{m}^{-2} \mathrm{sr}^{-1} \mathrm{sec}^{-1} \mathrm{GV}^{-1}, \gamma=-2.849 \pm 0.002$ (fit ${ }_{-0.003}^{+0.004}(\mathrm{sys})_{-0.003}^{+0.004}(\mathrm{sol}), \Delta \gamma=0.133_{-0.021}^{+0.032}(\mathrm{fit})_{-0.030}^{+0.046}$ (sys) \pm 0.005 (sol), $s=0.024_{-0.013}^{+0.020}(\mathrm{fit})_{-0.016}^{+0.027}(\mathrm{sys})_{-0.004}^{+0.006}(\mathrm{sol})$, and $R_{0}=336_{-44}^{+68}(\mathrm{fit})_{-28}^{+66}(\mathrm{sys}) \pm 1(\mathrm{sol}) \mathrm{GV}$. The first error quoted (fit) takes into account the statistical and uncorrelated systematic errors from the flux reported in [18]. The second (sys) is the error from the remaining systematic errors, namely from the rigidity resolution function and unfolding, and from the absolute rigidity scale, with their bin-to-bin correlations accounted for using the migration matrix $M_{i j}$. The third (sol) is the uncertainty due to the variation of the solar potential $\phi=0.50$ to $0.62 \mathrm{GV}$ [26]. The fit confirms that above $45 \mathrm{GV}$ the flux is incompatible with a single spectral index at the $99.9 \%$ C.L. The fit is shown in Fig. 3a.
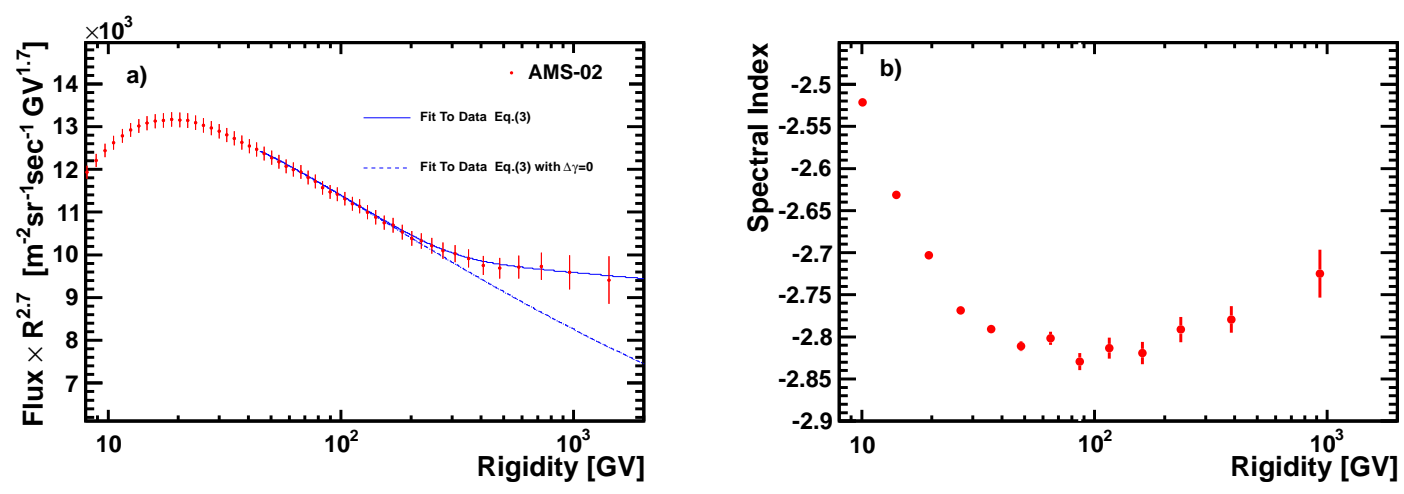

Figure 3: a) The AMS proton flux multiplied by $\tilde{R}^{2.7}$ as a function of rigidity $R$. The solid curve indicates the fit of Eq. 5.2 to the data. For illustration, the dashed curve uses the same fit values but with $\Delta \gamma$ set to zero. b) The dependence of the proton flux spectral index $\gamma$ on rigidity $R$.

For illustration, the fit results with $\Delta \gamma$ set to zero are also shown in Fig. 3 a.

To obtain the detailed variation of $\gamma$ with rigidity in a model independent way, the spectral index is calculated from

$$
\gamma=d[\log (\Phi)] / d[\log (R)]
$$


over independent rigidity intervals above $8.48 \mathrm{GV}$, with a variable width to have sufficient sensitivity to determine $\gamma$. The results are presented in Fig. 3b. As seen in Fig. 3 b, the spectral index varies with rigidity. In particular, the spectral index is progressively harder with rigidity above $\sim 100 \mathrm{GV}$.

In conclusion, knowledge of the rigidity dependence of the proton flux is important in understanding the origin, acceleration, and propagation of cosmic rays. Previous measurements of the proton flux in cosmic rays have reported different variations of the flux with energy and this has generated many theoretical models based on different sources, acceleration mechanisms, diffusive propagation effects, and their superposition. Our precise measurement of the proton flux from $1 \mathrm{GV}$ to $1.8 \mathrm{TV}$ is based on 300 million events and detailed studies of the systematic errors. The flux deviates from a single power law and the spectral index is progressively hardening at high rigidities.

\section{References}

[1] See, for example, P. Blasi, Braz. J. Phys. 44, 426 (2014); also P. Blasi, Astron. Astrophys. Rev., 21, 70 (2013).

[2] M. Ackermann et al., Phys. Rev. Lett. 112, 151103 (2014); M. Hareyama et al., J. Phys. Conf. 31, 159 (2006); M. Boezio et al., Astropart. Phys. 19, 583 (2003), E. Diehl, D. Ellithorpe, D. Muller, S.P. Swordy, Astropart. Phys. 18, 487 (2003); J. Alcaraz et al., Phys. Lett. B 490, 27 (2000); W. Menn et al., Astrophys. J. 533, 281 (2000); R. Bellotti et al., Phys. Rev. D 60, 052002 (1999); M. Boezio et al., Astrophys. J. 518, 457 (1999); K. Asakimori et al., Astrophys. J. 502, 278 (1998); M. Ichimura et al., Phys. Rev. D 48, 1949 (1993) I. P. Ivanenko et al., in Proceedings of the 23rd International Cosmic Ray Conference, Calgary, 17 (1993).

[3] For the ATIC experiment see A. D. Panov et al., Bull. Russian Acad. Sci. 73, 564 (2009) extracted from D. Maurin, F. Melot, R. Taillet, Astron. Astrophys. 569, A32 (2014)

[4] For a description of the BESS-Polar experiment see K. Abe et al., Phys. Rev. Lett. 108, 051102 (2012), For the BESS experiment see Y. Shikaze et al., Astropart. Phys. 28, 154 (2007); S. Haino et al., Phys. Lett. B 594, 35 (2004), T. Sanuki et al., Astrophys. J. 545, 1135 (2000).

[5] For the CREAM experiment see Y. S. Yoon et al., Astrophys. J. 728, 122 (2011)

[6] For the PAMELA experiment see O. Adriani, et al., Astrophys. J. 765, 91 (2013); O. Adriani, et al., Science 332, 69 (2011).

[7] A. Kounine, Int. J. Mod. Phys. E 211230005 (2012); S. Rosier-Lees, in Proceedings of Astroparticle Physics TEVPA/IDM, Amsterdam, 2014 (to be published); S. C. C. Ting, Nucl. Phys. B, Proc. Suppl. 243-244, 12 (2013); S.-C. Lee, in Proceedings of the 20th International Conference on Supersymmetry and Unification of Fundamental Interactions (SUSY 2012), Beijing, 2012 (unpublished); M. Aguilar, in Proceedings of the XL International Meeting on Fundamental Physics, Centro de Ciencias de Benasque Pedro Pascual, 2012 (unpublished); S. Schael, in Proceedings of the 10th Symposium on Sources and Detection of Dark Matter and Dark Energy in the Universe, Los Angeles, 2012 (unpublished); B. Bertucci,Proc. Sci. EPS-HEP, 67 (2011); M. Incagli, AIP Conf. Proc. 1223, 43 (2010), R. Battiston, Nucl. Instrum. Methods Phys. Res., Sect. A 588, 227 (2008).

[8] K. Luebelsmeyer et al., Nucl. Instrum. Methods Phys. Res., Sect. A 654, 639 (2011).

[9] B. Alpat et al., Nucl. Instrum. Methods Phys. Res., Sect. A 613, 207 (2010),

[10] V. Bindi et al., Nucl. Instrum. Methods Phys., Sect. A 743, 22 (2014) and references therein. 
[11] J. Allison et al., IEEE Trans. Nucl. Sci. 53, 270 (2006), S. Agostinelli et al., Nucl. Instrum. Methods Phys. Res., Sect. A 506, 250 (2003)

[12] J. Alcaraz et al., Phys. Lett. B 484, 10 (2000); see also Ref. [15].

[13] C. C. Finlay et al. Geophys. J. Int. 183/3, 1216 (2010).

[14] P. Papini et al., Astrophys. J. 615, 259 (2004)

[15] M. Aguilar et al., Physics Reports 366/6, 331 (2002)

[16] The AMS Collaboration, Measurement of the Flux of Helium Nuclei in Primary Cosmic Rays with the Alpha Magnetic Spectrometer on the International Space Station, (to be published); the AMS Collaboration, Measurement of the Flux of Light Nuclei in Primary Cosmic Rays with the Alpha Magnetic Spectrometer on the International Space Station, (to be published).

[17] Phys. Rev. Lett. 113, 121102 (2014)

[18] M. Aguilar et al., Phys. Rev. Lett. 114, 171103(2015);

[19] IGRF-12 (2015), currently available at http://www.ngdc.noaa.gov/IAGA/vmod/igrf.html (unpublished).

[20] P. Bobik, G. Boella, M. J. Boschini, D. Grandi, M. Gervasi, K. Kudela, S. Pensotti, P. G. Rancoita, Journal of Geophysical Research 111, A05205 (2006); N. A. Tsyganenko, M. I. Sitnov, Geophysical Res.: Space Physics 110, A3, 2156 (2005).

[21] N. Abgrall et al. Phys. Rev. C 84, 034604 (2011); J. R. Letaw, R. Silberberg, C. H. Tsao, Astrophys. J. Supp. 51271 (1983); A. S. Carroll et al., Phys. Lett. B 80, 319 (1979); S. P. Denisov, S. V. Donskov, Yu. P. Gorin, R. N. Krasnokutsky, A. I. Petrukhin, Yu. D. Prokoshkin, D. A. Stoyanova, Nucl. Phys. B 61, 62 (1973); G. Bellettini, G. Cocconi, A. N. Diddens, E. Lillethun, G. Matthiae, J. P. Scanlon, A. M. Wetherell, Nucl. Phys. 79, 609 (1966), T. Bowen, M. Di Corato, W. H. Moore, G. Tagliaferri, Il Nuovo Cimento 9, 908 (1958).

[22] L. Accardo et al., Phys. Rev. Lett. 113, 121101 (2014); M. Aguilar et al., Phys. Rev. Lett. 110, 141102 (2013). See Supplemental material at [URL will be inserted by publisher] for the tabulated flux.

[23] A progress report on our proton analysis was presented at the 33rd International Cosmic Ray Conference (2013). At that time our understanding of the systematic errors did not allow an accurate determination of the behavior of the proton flux.

[24] Nucl. Instr. Methods Phys. Res., Sect. A 355, 541 (1995) . We have used Eq. (6) with $\tilde{R} \equiv x_{l w}$.

[25] L. J. Gleeson, W. I. Axford, Astrophys. J. 154, 1101 (1968)

[26] I. G. Usoskin, G. A. Bazilevskaya, G. A. Kovaltsov, J. Geophys. Res. 116 A02104 (2011); K. G. McCracken, J. Beer, J. Geophys. Res. 112 A10101 (2007); I. G. Usoskin, K. Alanko-Huotari, G.A. Kovaltsov,K. Mursula, J. Geophys. Res. 110 A12108 (2005); 